\title{
La géographie en action dans les politiques de lutte contre la pauvreté en Pologne
}

Cet article est la version auteur d'un chapitre d'ouvrage dont la référence est :

Coudroy de Lille L., 2014, « La géographie en action dans les politiques de lutte contre la pauvreté en Pologne » in Boulineau E., Bonerandi-Richard E., La pauvreté en Europe, Rennes, Presses Universitaires de Rennes, pp. 187-204.

\section{Plan du texte}

Introduction.... 2

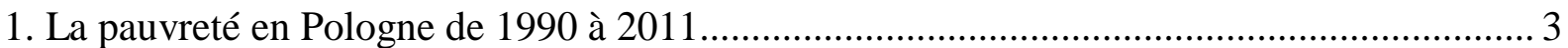

1.1. Convergence et restructuration économiques depuis les années $1990 \ldots \ldots \ldots \ldots \ldots \ldots \ldots \ldots \ldots . . . . . . . . . . .3$

1.2. La mesure de la pauvreté : l'européanisation des indicateurs ..................................... 3

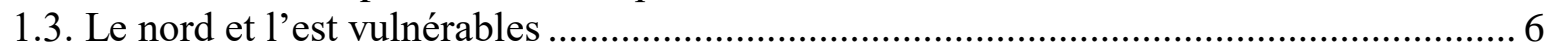

2. Les politiques étatiques de lutte contre la pauvreté.......................................................... 8

2.1. Les politiques nationales dans l'agenda communautaire ............................................. 8

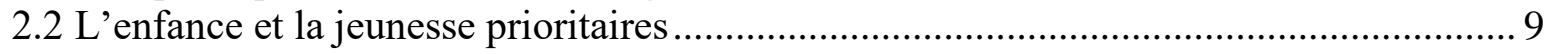

2.3. La dimension spatiale dans les documents des politiques publiques ........................... 9

3. Łódź au défi des quartiers insalubres et de la pauvreté des enfants ................................ 11

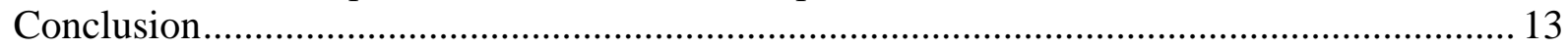

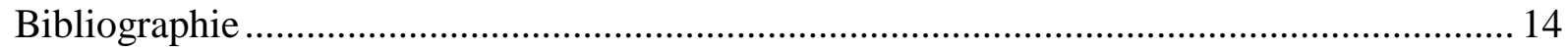

\section{Table des tableaux :}

Tableau 1 : Structure du PIB et de l'emploi en Pologne en1989 et en $2011 \ldots \ldots \ldots \ldots \ldots \ldots . . . . . . .3$

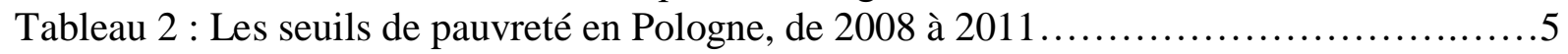

Tableau 3 : Les taux de risque de pauvreté dans les voïvodies en $2011(\%) \ldots \ldots \ldots \ldots \ldots \ldots . . . \ldots 7$

Tableau 4 : La dimension spatiale dans les documents de pilotage gouvernementaux........10

Table des figures

Figure 1 : Pauvreté, chômage et croissance économique en Pologne de 1993 à 2011 .............. 5

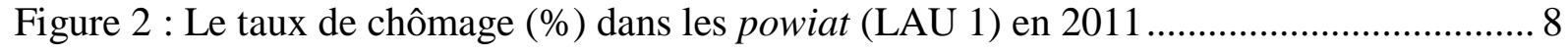

Figure 3 : Nombre de places dans les maisons à caractère social et salaire moyen dans les sous-régions (NUTS 3) en Pologne en 2009 ..................................................................... 11 


\section{Introduction}

État intermédiaire entre l'Europe de l'ouest et de l'est, inscrite dans une trajectoire économique de convergence progressive vers la moyenne de l'Union européenne, la Pologne occupe une position ambivalente vis-à-vis de la pauvreté, selon le point de vue adopté. Plus de deux millions Polonais ont afflué au Royaume-Uni et en Irlande en quête de meilleurs salaires, depuis l'ouverture en 2004 des marchés du travail de ces pays aux travailleurs de l'est. Mais si l'on se place de l'autre côté de la frontière orientale de la Pologne, la situation paraît bien différente. Pendant la campagne des élections législatives de 2012 en Ukraine, un des candidats se lamentait : «1000 euros de salaire moyen en Pologne, trois fois moins chez nous $! \gg{ }^{1}$. Du reste en Pologne, une partie des emplois peu qualifiés est désormais occupée par des immigrés, dont des Ukrainiens. Plaçons le regard alors en Pologne même.

Quelles sont les caractéristiques de la pauvreté dans cet État post-socialiste, entré dans la mondialisation dans les années 1990 et membre de l'UE depuis 2004 ? Premier constat : la pauvreté n'est pas une préoccupation scientifique et politique nouvelle, née des transformations systémiques des années 1990. Des études avaient été menées sur le sujet dans les années 1980 (Beskid, 198 ; Frąckiewicz, 1983 ; Jarosz, 1984) et montraient qu'à la fin de la décennie, environ $6 \%$ de la population était pauvre, c'est-à-dire vivait avec moins du minimum vieillesse. Un ouvrage français consacré aux inégalités en Europe de l'est à cette époque, faisait écho à ces thèmes de réflexion (Kende et Strmiska, 1984). Deuxièmement, la Pologne est le berceau de la «thérapie de choc » décrite plus loin. On peut donc la considérer comme un laboratoire de transformation de la pauvreté dans un contexte de passage accéléré au libéralisme économique; le secrétaire général de l'équipe ayant élaboré la Stratégie nationale soulignait d'ailleurs que la spécificité de la pauvreté en Pologne résidait dans la conjugaison d'héritages sociaux liés de l'ancien régime avec la brutalité de comportements d'acteurs économiques «à l'américaine » (Miżejewski, 2005). Enfin sur une superficie de $312000 \mathrm{~km}^{2}$, les disparités géographiques sont incontournables. Les indicateurs de convergence économique mesurée au niveau étatique doivent en permanence être déclinés au niveau régional, voire infrarégional. Se poser la question des formes nouvelles de la pauvreté consiste donc à interroger le déplacement de ses frontières sociales et spatiales. À cet égard, le niveau territorial de la région, voire du local sera ici considéré non pas seulement comme une échelle d'observation, mais également d'action, car la Pologne est devenue un État décentralisé, déléguant des compétences économiques et sociales aux niveaux infra-étatiques. L'analyse qui est présentée ici insistera sur la dimension spatiale à la fois des manifestations de la pauvreté en Pologne, et des politiques qui sont menées contre son extension, dans la perspective de l'européanisation des politiques publiques. Dans un pays où la conscience des inégalités spatiales de développement imprègne le discours politique depuis l'entre-deuxguerres, et où les outils d'une politique multi-niveaux existent, on peut faire l'hypothèse que cette dimension spatiale est réelle, ce que nous chercherons à valider ou à nuancer. La mise en place de politiques communautaires coordonnées à partir des années 2000 a-t-elle enrichi ou au contraire appauvri la dimension spatiale de la lutte contre la pauvreté ? Quel rôle jouent les niveaux spatiaux infra-étatiques, quelles relations ceux-ci entretiennent-ils avec l'État et l'Union? Une ville pilote dans les programmes de lutte contre la pauvreté sera mise en avant comme exemple.

\footnotetext{
${ }^{1}$ «Vitali Klitchko, un « cogneur » aux législatives en Ukraine », Le Monde, 28-29 octobre 2012.
} 


\section{La pauvreté en Pologne de 1990 à 2011}

\subsection{Convergence et restructuration économiques depuis les années 1990}

La Pologne est inscrite dans une trajectoire de convergence économique dans l'Union Européenne : le PIB/habitant en SPA était de $43 \%$ de la moyenne européenne (UE 27) en 1995 et $65 \%$ en 2011 ; c'est le seul État de l'UE dont le taux de croissance est resté positif dans les années 2000, y compris au plus fort de la crise financière (4,3\% en 2011). L'économie est néanmoins en pleine mutation depuis une vingtaine d'années (tableau 1) : si l'agriculture emploie encore $12 \%$ de la population active, la tertiarisation est massive, sans toutefois faire de la Pologne un pays «post-industriel», surtout si on le compare à la moyenne européenne : un peu moins d'un tiers de la production et de l'emploi dépendent encore de l'industrie. Les branches de l'industrie mécanique (automobile), du bois (meuble), des chantiers navals, de la métallurgie associée aux mines sont les points forts de l'industrie polonaise qui est spatialement concentrée dans le sud (Haute et Basse Silésie), les grandes agglomérations, et les ports de la Baltique.

Tableau 5 : Structure du PIB et de l'emploi en Pologne en1989 et en 2011

\begin{tabular}{|l|c|c|c|c|}
\hline \multirow{2}{*}{$\%$} & \multicolumn{2}{|c|}{ PIB } & \multicolumn{2}{c|}{ Population active } \\
\cline { 2 - 5 } & 1989 & 2011 & 1989 & 2011 \\
\hline Agriculture & 14 & 4 & 28 & 12 \\
\hline Industrie & 61 & 30 & 37 & 31 \\
\hline Services & 25 & 66 & 35 & 57 \\
\hline
\end{tabular}

Coudroy 2013, UMR CNRS EVS. Source : Office central de statistique (GUS), 2012

Ces transformations structurelles de l'économie sont le résultat notamment d'une évolution inaugurée par la «thérapie de choc » dès la chute du régime socialiste, suivie de l'adaptation à la mondialisation et de la préparation à l'intégration européenne. Conceptualisée par l'économiste américain libéral Jeffrey Sachs et mise en œuvre par le ministre des finances Leszek Balcerowicz, la thérapie de choc consista d'une part à libérer simultanément les prix, la création d'entreprises et le choix des partenaires commerciaux, d'autre part, à privatiser de larges pans de l'économie socialiste, enfin à libéraliser les échanges de devises (Colas, 2002). Elle se traduisit par la disparition soudaine de la pénurie dans les magasins, mais aussi et surtout par une hyperinflation (plus de $600 \%$ en 1989, $250 \%$ en 1990, $60 \%$ en 1991), le démantèlement des grandes entreprises d'État, et par conséquent l'apparition puis le maintien d'un chômage structurel. Le taux de chômage passa de $0 \%$ en 1989 à $15 \%$ dès 1992, pour osciller entre 10 et $20 \%$ depuis les années 1990, malgré la reprise économique après 1992. L'économie s'est largement européanisée et ouverte aux investissements étrangers, mais à l'échelle de l'UE-27, la Pologne demeure un pays économiquement pauvre: dans le classement du PIB/habitant (en SPA), elle occupait le $24^{\mathrm{e}}$ rang à la veille de l'intégration (2003) et le $23^{\mathrm{e}}$ en $2010^{2}$. Les deux dernières décennies ont enfin été marquées par une légère augmentation de la population (+0,4\% depuis 1990), ce qui distingue la Pologne de ses voisins, dont plusieurs ont connu une déprise démographique profonde. L'émigration est peu visible statistiquement car il s'agit souvent de mobilités circulatoires au sein de l'espace Schengen. Dans ce contexte, la pauvreté a changé de visages, et ses indicateurs également.

\subsection{La mesure de la pauvreté : l'européanisation des indicateurs}

La langue polonaise est riche pour nommer la pauvreté ! On compte dans la langue courante pas moins de quatre mots quasiment synonymes (bieda, ubóstwo, niezamożność, niedostatek),

\footnotetext{
${ }^{2}$ Les indicateurs socio-économiques proviennent d'Eurostat, de l'office central de statistiques polonais (le GUS) et de la CNUCED pour les IDE.
} 
définis par le dictionnaire comme «le manque de moyens matériels suffisants $»^{3}$; la langue scientifique ou d'expertise n'a pas tranché, utilisant l'un ou l'autre de ces termes, avec une préférence pour les deux premiers, et s'est enrichie de néologismes issus de la commande européenne, comme les indicateurs lejkenowskie (de Laeken).

Comme indiqué plus haut, la Pologne n'a pas découvert la thématique de la pauvreté après 1990, mais les indicateurs qu'elle utilise ont varié à mesure de l'européanisation du traitement de la pauvreté. Il faut rappeler que les échanges scientifiques et intellectuels internationaux avaient perduré pendant la période socialiste, si bien que les sciences sociales jouissaient d'une vivacité supérieure à ce qui pouvait exister dans les autres pays du bloc de l'est, toutes choses égales par ailleurs. La décennie 1980-89 voit donc culminer à la fois les problèmes politiques et socio-économiques (avec l'état de guerre décrété en décembre 1981) et les travaux critiques sur l'état du pays. C'est ainsi qu'en 1981 est mis au point l'indicateur du minimum social (minimum socjalne) et le minimum vital (minimum egzystencji) par l'Institut du Travail et des Affaires Sociales. Le premier correspond à la valeur d'un panier de biens élémentaires permettant une vie sociale minimale, incluant une participation à la vie culturelle et récréative. Le second se réduit à un panier de biens assurant la survie biologique du ménage, excluant donc la culture et les loisirs. Ces deux indicateurs sont évalués en continu depuis 1981, avec des adaptations annuelles à l'état du marché (Baczewski, 2008). La valeur de ce panier de biens est également différenciée régionalement (elle varie jusqu'à plus ou moins $5 \%$ de la moyenne nationale). Mais le minimum social est jugé aujourd'hui comme étant une limite trop élevée pour mesurer efficacement la pauvreté ${ }^{4}$. Il reste utilisé pour définir la pauvreté légale (ubóstwo ustawowe), celle qui déclenche l'obtention de prestations sociales. En revanche le minimum vital est toujours utilisé pour définir la pauvreté absolue ou extrême (ubóstwo absolutne, skrajne). C'est le seul exemple de continuité dans les instruments de mesure de la pauvreté.

En effet, les transformations systémiques intervenues après 1989 constituent une rupture telle qu'elle obère toute comparaison diachronique appuyée sur des bases de donnés socioéconomiques. Restent les grandes enquêtes. Un sociologue a tenté de mesurer l'évolution de la privation matérielle pendant le communisme et après, dans six pays, en posant des questions simples à des personnes de plusieurs générations, relatives à leur situation à l'âge de 14 ans, comme : leur arrivait-il d'aller se coucher en ayant faim ? Avaient-elles une paire de chaussures solides de rechange ? Un vêtement chaud pour l'hiver ? Il en ressort que pendant toute la période communiste, la pauvreté a diminué partout de manière très significative, et qu'elle a augmenté dans les années 1990 et jusque dans les années 2000 (Domański, 2002),. Ainsi apparaît un premier paradoxe, que la Pologne partage avec les autres pays de l'ancien bloc socialiste : la libéralisation économique s'est traduite par l'augmentation simultanée de la production de richesses (illustrée par la convergence) et de la paupérisation. C'est pourquoi la pauvreté dérange dans le spectre politique post-1990 à la fois les partis ayant incarné la transition (car la pauvreté s'est accentuée avec leur arrivée au pouvoir) et les partis postcommunistes (car il est désormais admis qu'elle est partiellement héritée du régime précédent). À partir des années 2000, les comparaisons synchroniques se multiplient, utilisant les données initiées par la communauté européennes (EU-SILC). L’homogénéisation des indicateurs accompagne ce mouvement.

L'indicateur de la pauvreté relative (ubóstwo relatywna) dite aussi monétaire (ubóstwo dochodowy) est apparu avec la mise en place des politiques européennes; il est égal en Pologne à $60 \%$ de la médiane des revenus des ménages de structure équivalente, selon les recommandations de l'UE. La pauvreté subjective est également utilisée, parfois en lieu et place de la pauvreté absolue.

\footnotetext{
3 Définitions consultées sur le dictionnaire de la langue polonaise des éditions scientifiques PWN (http://sjp.pwn.pl/). Toutes les traductions du polonais au français dans le reste du texte sont de l'auteur.

${ }^{4} 58 \%$ de la population en 2003 vivait avec des revenus inférieurs au minimum social, le GUS a renoncé à poursuivre cette évaluation après 2004. Mais le minimum social est encore utilisé pour définir la pauvreté légale.
} 
Tableau 6 : Les seuils de pauvreté en Pologne, de 2008 à 2011

\begin{tabular}{|c|c|c|c|c|c|c|}
\hline & \multicolumn{2}{|c|}{ Pauvreté absolue } & \multicolumn{2}{c|}{ Pauvreté relative } & \multicolumn{2}{c|}{ Pauvreté légale } \\
\hline & Złoty & $€$ & Złoty & $€$ & Złoty & $€$ \\
\hline 2008 & 418 & 119 & 612 & 175 & 477 & 136 \\
\hline 2009 & 443 & 105 & 633 & 151 & 477 & 114 \\
\hline 2010 & 466 & 119 & 665 & 171 & 477 & 122 \\
\hline 2011 & 495 & 121 & 690 & 168 & 477 & 116 \\
\hline
\end{tabular}

Coudroy 2013, UMR CNRS EVS. Sources : GUS et Banque nationale de Pologne pour les conversions de change annuels moyens.

Comme on le voit sur le tableau 2, le seuil de pauvreté légale stagne (il est à ce niveau de 477 złoty depuis 2006), tandis que l'inflation a affecté les autres seuils qui ont été revus à la hausse. On est arrivé à un stade critique où le seuil de pauvreté absolu a dépassé en 2011 le niveau légal, de sorte qu'une personne touchant 495 złoty par mois n'est plus éligible aux prestations sociales.

Ces seuils concernent une part variable de la population. Le taux de risque de pauvreté relative s'élève ainsi à $16,7 \%$ de la population en 2011 , alors que le taux de pauvreté absolue n'en concerne que $6,5 \%$. Globalement, la pauvreté mesurée par ces indicateurs avait augmenté pendant les années 1990, et la figure 1 montre la poursuite de cette tendance jusqu'au renversement des années 2003-2005 : les effets socio-économiques de l'intégration européenne sont donc réels ${ }^{5}$. La pauvreté absolue a connu l'évolution la plus heurtée, augmentant continuellement pendant les années 1990, pour décliner à partir de 2005, puis remonter légèrement depuis 2009.

Figure 1 : Pauvreté, chômage et croissance économique en Pologne de 1993 à 2011

(Population vivant dans des ménages relevant des différents types de pauvreté, taux de chômage, taux de croissance du PIB, en \%).

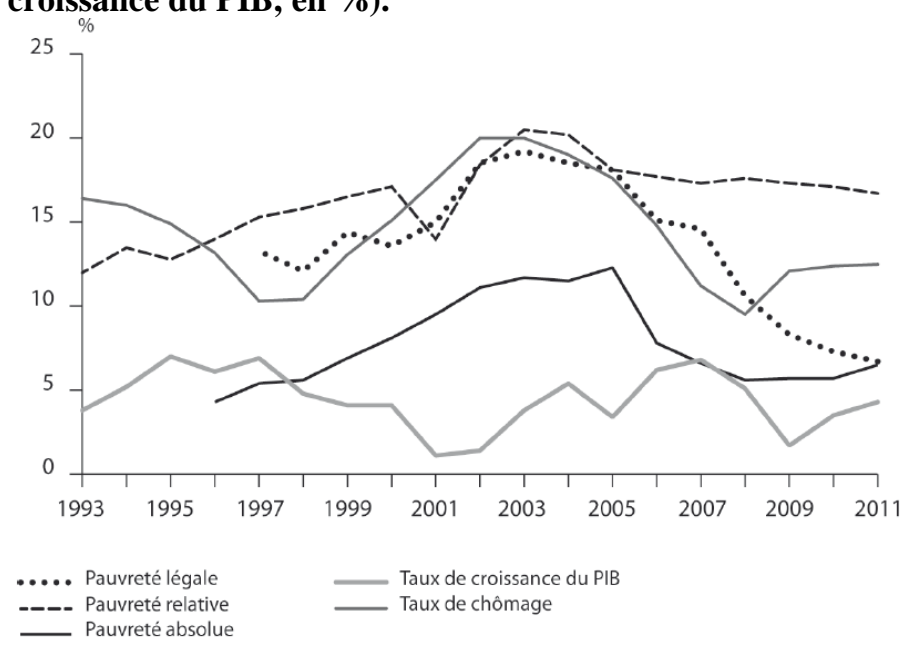

Coudroy 2013, UMR CNRS EVS. Source : GUS, 2012.

Néanmoins le document sur lequel sont fondées les politiques de lutte contre la pauvreté dans les années 2000, la Stratégie Nationale pour l'inclusion sociale, retient comme notion cardinale celle d'exclusion sociale (wykluczenie społeczne). Elle y est définie comme une « situation rendant impossible ou très difficile à un individu ou à un groupe l'accomplissement de son rôle social, l'accès aux biens publics et aux infrastructures sociales, l'accumulation de ressources et de revenus dignes $»^{6}$. Les auteurs expliquent en effet que l'on

\footnotetext{
${ }^{5}$ Entre 2003 et 2007 le taux de croissance de l'économie polonaise est passé de 3,8 à 6,8\%.

6 «Wykluczenie społeczne to sytuacja uniemożliwiająca lub znacznie utrudniająca jednostce lub grupie, zgodne z prawem pełnienie ról społecznych, korzystanie z dóbr publicznych i infrastruktury społecznej, gromadzenie zasobów i zdobywanie dochodów w godny sposób » (Golinowska 2004, p. 23).
} 
peut être exclu mais pas pauvre, et inversement, que l'on peut relever de la pauvreté relative sans être exclu ; ils opposent ainsi à l'exclusion un programme d'inclusion (inkluzja) et de cohésion (spójność).

Les catégories sociales les plus exposées ne sont pas toujours celles que l'on attend. Les familles ne vivant que des transferts sociaux sont les plus vulnérables face à la pauvreté absolue $(30 \%)$. Puis viennent les chômeurs (le taux de pauvreté absolue est de 12,5\% dans les ménages comptant un chômeur en 2011, soit le double de la moyenne nationale, contre $5 \%$ là où il n'y en a aucun). Mais les travailleurs pauvres, nouvelle catégorie sociale apparue avec l'économie libérale, ne sont guère moins exposés. Malgré les succès de la Pologne dans la politique agricole commune, les agriculteurs sont touchés de la même manière que les chômeurs; il s'agit des petits paysans à la tête de micro-exploitations. Enfin les retraités, à rebours de l'opinion générale, ne sont pas les plus vulnérables (4,5\% de pauvreté absolue).

La structure familiale est un facteur discriminant également important. En 2011 toujours, $10,5 \%$ des enfants vivent dans des familles relevant de la pauvreté absolue (contre $6,5 \%$ de la population globale) car les familles nombreuses sont les plus fragiles - davantage que les familles monoparentales. En conséquence, $31 \%$ de la population frappée par la pauvreté absolue est constitué de mineurs, alors que les enfants de moins de 18 ans ne représentent que $20 \%$ de la population totale.

\subsection{Le nord et l'est vulnérables}

Les études menées par l'Office central de statistiques, le GUS, soulignent la plupart du temps non seulement la plus ou moins grande vulnérabilité des groupes sociaux, mais aussi de certains types d'espaces. Trois grands types d'oppositions spatiales en ressortent : d'une part, le contraste ville/campagne : 4,2\% des citadins vivaient en 2011 dans des ménages relevant de la pauvreté absolue, contre 10,9\% des ruraux (Szukiełojć-Biekuńska, 2011b). La pauvreté rurale est maximale dans les régions septentrionales, où elle est l'expression d'un chômage agricole né du démantèlement des exploitations collectives qui y étaient cantonnées (figure 2). La Pologne comptant encore presque un tiers de population rurale, $60 \%$ des pauvres (pauvreté absolue) vivent dans les campagnes. D'autre part, les habitants des grandes villes sont nettement favorisés par rapport à ceux des petites villes, et au sein des villes, la polarisation socio-spatiale augmente (Węcławowicz, 2001, 2003). Enfin régionalement, les contrastes opposent les régions de l'est peu urbanisées et peu industrialisées (VarmieMazurie, Podlasie, voïvodie de Lublin toutes deux à $11 \%$ environ) aux régions occidentales (3,2 \% dans la voïvodie de Lubusz), méridionales (entre 4 et $5 \%$ dans les régions très urbaines et industrielles de Silésie, Petite Pologne) et bien sûr de la Mazovie où siège la capitale $(4,6 \%)$. Les sous-régions où le salaire moyen est le plus élevé apparaissant sur la figure 3 sont à la fois les métropoles et les régions minières de Haute et Basse Silésie. 
Tableau 7 : Les taux de risque de pauvreté dans les voïvodies en 2011 (\%)

\begin{tabular}{|c|c|c|c|}
\hline $\begin{array}{l}\text { Nom polonais } \\
\text { Nom français }\end{array}$ & $\begin{array}{l}\text { Pauvreté } \\
\text { absolue }\end{array}$ & Pauvreté relative & $\begin{array}{c}\text { Pauvreté } \\
\text { légale }\end{array}$ \\
\hline $\begin{array}{c}\text { Warmińsko-Mazurskie } \\
\text { Varmie-Mazurie }\end{array}$ & 11,2 & 24,2 & 10,2 \\
\hline $\begin{array}{l}\text { Podlaskie } \\
\text { Podlasie }\end{array}$ & 11 & 23,9 & 10,1 \\
\hline $\begin{array}{c}\text { Lubelskie } \\
\text { Vö̈vodie de Lublin }\end{array}$ & 10,9 & 23,1 & 10,2 \\
\hline $\begin{array}{l}\text { Świętokrzyskie } \\
\text { Sainte-Croix }\end{array}$ & 10 & 26,7 & 9 \\
\hline $\begin{array}{c}\text { Wielkopolskie } \\
\text { Grande Pologne }\end{array}$ & 9,2 & 20,9 & 9,1 \\
\hline $\begin{array}{l}\text { Pomorskie } \\
\text { Poméranie }\end{array}$ & 9,1 & 19,4 & 9,8 \\
\hline $\begin{array}{c}\text { Zachodniopomorskie } \\
\text { Poméranie Occidentale }\end{array}$ & 7,5 & 17,6 & 7,3 \\
\hline $\begin{array}{l}\text { Kujawsko-Pomorskie } \\
\text { Coujavie-Poméranie }\end{array}$ & 7,4 & 18,1 & 6,9 \\
\hline $\begin{array}{c}\text { Podkarpackie } \\
\text { Basses Carpates }\end{array}$ & 6,9 & 21,1 & 6,6 \\
\hline $\begin{array}{c}\text { Łódzkie } \\
\text { Vö̈vodie de Łódź }\end{array}$ & 5,6 & 13,1 & 6 \\
\hline $\begin{array}{c}\text { Małopolskie } \\
\text { Petite Pologne }\end{array}$ & 4,8 & 14,8 & 6,3 \\
\hline $\begin{array}{l}\text { Śląskie } \\
\text { Silésie }\end{array}$ & 4,8 & 12,3 & 4,8 \\
\hline $\begin{array}{l}\text { Dolnośląskie } \\
\text { Basse Silésie }\end{array}$ & 4,7 & 13,6 & 5,1 \\
\hline $\begin{array}{c}\text { Opolskie } \\
\text { Voüvodie d'Opole }\end{array}$ & 4,7 & 10,3 & 4,3 \\
\hline $\begin{array}{c}\text { Mazowieckie } \\
\text { Mazovie }\end{array}$ & 4,6 & 12,1 & 4,2 \\
\hline $\begin{array}{c}\text { Lubuskie } \\
\text { Vö̈vodie de Lubusz }\end{array}$ & 3,2 & 13,4 & 3 \\
\hline Pologne & 6,5 & 16,7 & 6,7 \\
\hline
\end{tabular}

Coudroy 2013, UMR CNRS EVS. Source : GUS, 2012.

Ces différenciations spatiales ne sont que partiellement l'expression d'une "géographie des transformations structurelles $»$ : elles ont un ancrage historique profond, qui imprime le territoire polonais depuis la fin du XVIII ${ }^{\mathrm{e}}$ siècle. Les régions de l'est étaient sous domination russe lorsque la Pologne fut rayée de la carte et partagée entre les empires européens, alors que celles de l'ouest et du sud qui étaient sous domination prussienne et autrichienne en sont sorties plus industrialisées, plus modernes, plus urbanisées. Le socialisme et les logiques économiques post-socialistes ont exacerbé cet héritage géopolitique. A une échelle fine, comme l'indique la carte du chômage (fig. 2), la prospérité relative des grandes villes, îlots de plein emploi parfois au sein de régions durement éprouvées, apparaît nettement. 


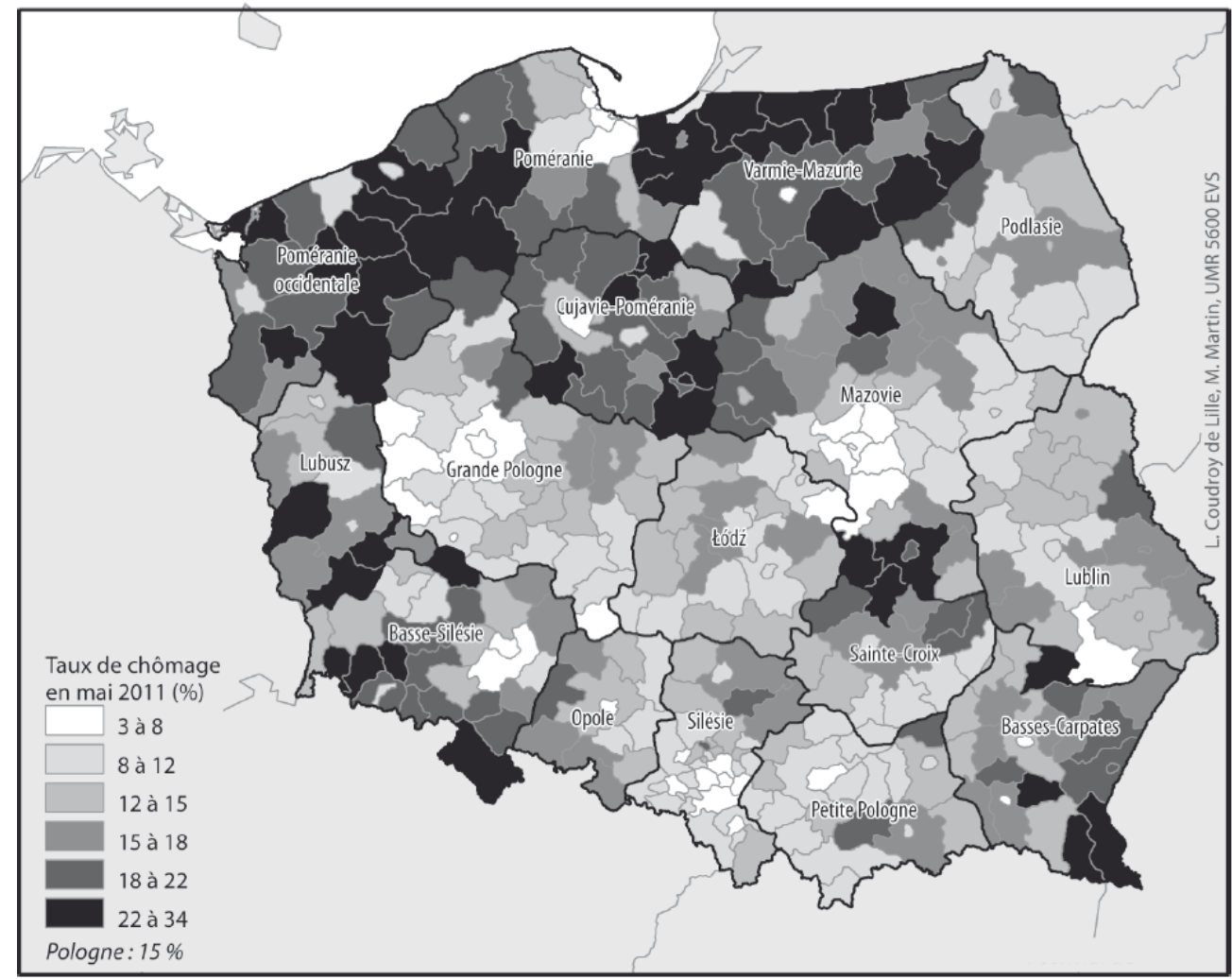

Coudroy 2013, UMR CNRS EVS. Source : GUS, 2012.

\section{Les politiques étatiques de lutte contre la pauvreté}

\subsection{Les politiques nationales dans l'agenda communautaire}

La Constitution de 1997 oblige l'État à lutter contre la pauvreté, et un certain nombre de lois adoptées dans les années 2000 ferment la première décennie post-socialiste marquée par la mise en place d'un capitalisme libéral aux effets très défavorables à certaines catégories sociales. Emergent alors des initiatives inspirées par l'État : ainsi des lois sont votées sur le salaire minimum en 2002, l'emploi social en 2003, l'aide sociale en 2004. D'autres proviennent du cadre européen, à l'instar du Mémorandum commun pour l'intégration sociale signé par la Pologne en 2003 et des deux documents qui lui sont liés : d'une part la Stratégie Nationale pour 1'Inclusion Sociale (SNIS) (Golinowska, 2004) ${ }^{7}$ qui fixait les objectifs à atteindre pour 2010, d'autre part, le Plan National d'actions pour les années 2004-06 (ND, 2004), préparé simultanément et transmis à l'UE dans le cadre de la Méthode ouverte de Coordination «Inclusion sociale ». Il indique comment les objectifs de la stratégie seront atteints. Il s'agit donc du premier Plan national d'action pour l'inclusion (PNAI). Pour élaborer ces deux documents, le gouvernement a constitué un groupe de travail de 32 personnes issues de l'administration d'État, des collectivités (voïvodies et powiat, c'est-à-dire des niveaux NUTS 2 et LAU 1), d'organisations internationales, d'ambassades, et de la société civile, essentiellement d'associations à caractère social et humanitaire. Deux autres PNAI suivront, l'un pour la période 2006-2008 (ND, 2006), l'autre pour 2008-2010 (ND, 2008). C'est ce dernier qui a été retenu et traduit en anglais pour la Commission, et mis en ligne. Entretemps, la Stratégie nationale a été reformulée en 2005 pour la période 2007-13, en vue de la réforme des retraites dont l'entrée en vigueur était prévue en 2009, et du plan national de développement (ND, 2005). En outre, la période 2007-2013 correspond également à la mise en place de la stratégie de cohésion comprenant le programme opérationnel «Capital humain » (Coudroy de Lille, 2009). Celui-ci occupe le $3^{\mathrm{e}}$ poste de dépenses parmi

\footnotetext{
${ }^{7}$ Nardowa Strategia Integracji Społecznej (NSIS)
} 
les aides structurelles reçues par la Pologne (13\%, soit 9707 millions euros), derrière les dépenses «Infrastructures et environnement » et les «programmes régionaux opérationnels ». Tous ces documents programmatiques se sont appuyés sur des rapports rédigés par le GUS qui, outre ses analyses récurrentes sur les conditions de vie et la pauvreté des Polonais (GUS, 1997, 2003, 2006, 2012 ; Szukiełojć-Biekuńska, 2011a, b), a rédigé des analyses basées sur les données UE-SILC (Łysoń, 2011 ; Szukiełojć-Biekuńska, 2010). A cela s'ajoutent les publications de deux institutions importantes dans le champ social : l'une, historique, l'Institut du Travail et des Affaires sociales (fondé en 1962), l'autre plus récente, la délégation polonaise du réseau Europe Against Poverty Network (EAPN).

\subsection{L'enfance et la jeunesse prioritaires}

La SNIS de 2004, premier document d'envergure de niveau national, commence par un diagnostic multidimensionnel de la pauvreté en Pologne, puis présente d'une part les groupes sociaux les plus vulnérables, d'autre part une liste de vingt actions prioritaires qui en découlent. Dans l'une et l'autre de ces rubriques se dégage une attention particulière portée vers les enfants, les mères célibataires, et les familles nombreuses. Parmi les groupes vulnérables suivent les personnes touchées par le chômage et/ou en manque de qualification, des groupes définis par leur situation sanitaire, résidentielle, et en fin de liste, les immigrants et les minorités roms (ces deux catégories de population étant peu nombreuses en Pologne). Logiquement, les priorités d'action reflètent ces hiérarchies, puisque six d'entre elles concernent les enfants et leur éducation. Tout cela se traduit dans le premier PNAI de 2004 par l'accent mis sur les actions éducatives et sociales destinées à la jeunesse, tout comme dans la Stratégie de 2005 et les PNAI de 2006 et 2008 qui ont une tonalité plus libérale, insistant davantage, pour les adultes, sur la notion de retour à l'emploi (aktywizacja).

\subsection{La dimension spatiale dans les documents des politiques publiques}

La dimension spatiale est partout présente dans les textes définissant les politiques publiques, qu'il s'agisse des documents stratégiques ou d'action (tableau 2). On peut la trouver dans les diagnostics réalisés sur la pauvreté et ses corollaires, mais aussi à travers les recommandations, lorsque celles-ci indiquent quels niveaux spatiaux peuvent être mobilisés, ou bien quels types d'espaces sont prioritaires. Elle irrigue enfin l'argumentaire à travers des graphiques ou tableaux rendant compte des inégalités spatiales (ne sont alors pris en compte dans le tableau 4 que ceux qui sont systématiques, présentant par exemple des données nationales déclinées pour toutes les régions, ou pour toutes les tailles de villes). 
Tableau 8 : La dimension spatiale dans les documents de pilotage gouvernementaux

\begin{tabular}{|c|c|c|c|c|c|c|}
\hline \multicolumn{2}{|c|}{ Titre } & $\begin{array}{c}\text { Stratégie } \\
\text { nationale } \\
\text { d'intégration } \\
\text { sociale pour la } \\
\text { Pologne }\end{array}$ & $\begin{array}{c}\text { Plan national } \\
\text { d'actions pour } \\
\text { l'intégration } \\
\text { sociale 2004- } \\
2006\end{array}$ & $\begin{array}{c}\text { Stratégie de la } \\
\text { politique } \\
\text { sociale pour les } \\
\text { années 2007-13 }\end{array}$ & $\begin{array}{l}\text { Programme } \\
\text { national } \\
\text { «Sécurité } \\
\text { sociale et } \\
\text { inclusion } \\
\text { sociale pour les } \\
\text { années 2006- } \\
2008\end{array}$ & $\begin{array}{l}\text { Programme } \\
\text { national } \\
\text { «Sécurité } \\
\text { sociale et } \\
\text { intégration } \\
\text { sociale pour les } \\
\text { années 2008- } \\
2010 »\end{array}$ \\
\hline \multicolumn{2}{|c|}{ Nombre de pages } & 90 & 72 & 56 & 121 & 154 \\
\hline \multicolumn{2}{|c|}{ Type de document } & Stratégie & PNAI & Stratégie & PNAI & PNAI \\
\hline \multicolumn{2}{|c|}{ Année } & 2004 & 2004 & 2005 & 2006 & 2008 \\
\hline \multirow{5}{*}{ Diagnostic } & Ville/campagne & $\mathrm{x}$ & $\mathrm{x}$ & $\mathrm{x}$ & $\mathrm{x}$ & $\mathrm{x}$ \\
\hline & Taille des villes & $\mathrm{x}$ & $\mathrm{x}$ & & $\mathrm{x}$ & $\mathrm{x}$ \\
\hline & $\begin{array}{c}\text { Inégalités inter- } \\
\text { régionales }\end{array}$ & $\mathrm{X}$ & $\mathrm{x}$ & $\mathrm{x}$ & $\mathrm{X}$ & $\mathrm{x}$ \\
\hline & $\begin{array}{c}\text { Inégalités } \\
\text { PL/PECO/UE15 }\end{array}$ & $\mathrm{x}$ & $\mathrm{X}$ & & & \\
\hline & $\begin{array}{c}\text { Déficit } \\
\text { d'infrastructures } \\
\text { (logement, } \\
\text { transports) }\end{array}$ & X & $X$ & $\mathrm{x}$ & $X$ & $\mathrm{X}$ \\
\hline \multirow[t]{2}{*}{ Recommandations } & $\begin{array}{l}\text { Espaces à } \\
\text { privilégier }\end{array}$ & & $\begin{array}{l}\text { Quartiers } \\
\text { urbains } \\
\text { dégradés }\end{array}$ & & & $\begin{array}{l}\text { campagnes } \\
500 \text { communes } \\
\text { les plus pauvres }\end{array}$ \\
\hline & $\begin{array}{c}\text { Implication des } \\
\text { collectivités }\end{array}$ & $\mathrm{x}$ & $\mathrm{X}$ & $\mathrm{X}$ & $\mathrm{x}$ & $\mathrm{x}$ \\
\hline Argumentaire & $\begin{array}{c}\text { Statistiques } \\
\text { spatiales } \\
\text { systématiques, } \\
\text { cartes }\end{array}$ & $X$ & & & & $\mathrm{x}$ \\
\hline
\end{tabular}

$\mathrm{x}=$ présence de l'information

$\mathrm{X}=$ développement de l'information

Coudroy 2013, UMR CNRS EVS. Sources : documents cités dans le tableau.

Les documents stratégiques sont à observer en priorité, puisqu'ils guident les PNAI. Sur ce point, la stratégie de 2004 recèle une vision spatiale beaucoup plus riche que celle de 2005 : c'est le seul document qui présente sous la forme de tableaux ou de graphiques le taux de risque de pauvreté selon les catégories de taille des villes, et selon la voïvodie (région, NUTS 2). Il compare également la Pologne aux autres nouveaux États membres (de 2004) et à l'UE-15. Dans leur diagnostic, tous ces documents convergent sur les inégalités spatiales opposant villes et campagnes, petites et grandes villes et les régions entre elles (voir point 1.3 supra). Seuls ceux rédigés en 2004 comparent la Pologne aux pays situés au même stade de l'intégration européenne. Les nuances les plus fortes dans les diagnostics concernent le rôle attribué aux déficiences dans l'équipement du territoire : certains mettent l'accent sur le manque de logements (Stratégie et PNAI de 2004, PNAI de 2006), d'autres sur le démantèlement du transport public et notamment des lignes ferroviaires secondaires (PNAI de 2006). Du côté des recommandations, tous font mention du rôle que doivent jouer les collectivités, c'est-à-dire les voïvodies, les powiat, et les communes.

Parfois, le niveau local est également sollicité du côté du «tiers secteur», à travers les associations locales et les coopératives, un secteur important historiquement en Pologne, dont le rôle est souligné dans la stratégie de 2005. L'Eglise, à travers son bras droit Caritas Polska qui est très solidement implanté dans ce pays, n'est citée qu'une seule fois dans la Stratégie de 2004, et cela dans la partie «bonnes pratiques »- mais elle est mentionnée dès l'article 2 de la loi sur l'aide sociale de 2004. Enfin, dans certains PNAI, l'espace est présent sous la forme 
d'une sorte de géographie prioritaire : les quartiers anciens dégradés pour le PNAI de 2004, les communes rurales les plus pauvres pour celui de 2008 .

Figure 3 : Nombre de places dans les maisons à caractère social et salaire moyen dans les sous-régions (NUTS 3) en Pologne en 2009

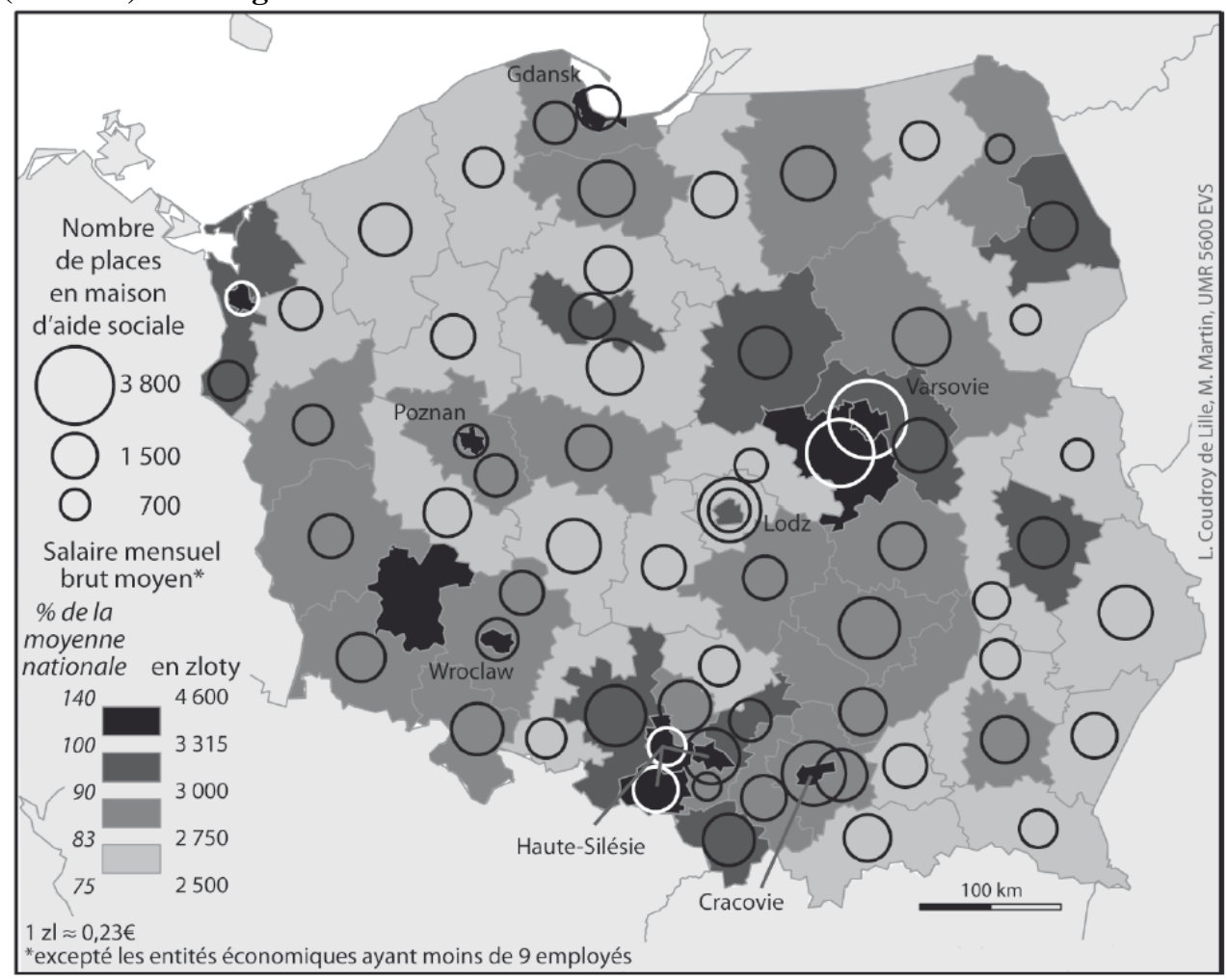

Coudroy 2013, UMR CNRS EVS. Source : GUS (Office central de statistiques), 2012.

La dimension spatiale souffre donc d'une certaine déperdition entre les documents stratégiques de 2004 et 2005, et d'un manque de cohérence entre les différents PNAI, ceux-ci insistant tour à tour sur certains aspects et pas d'autres. Aucun de ces documents ne comprend de carte, à la différence des rapports effectués par le GUS, alors même que ces derniers sont parfois très synthétiques, de l'ordre d'une dizaine de pages. Ainsi, même si certains documents utilisent des formules rodées et fortes, comme celle du «mur de l'Est » désignant les régions orientales les moins bien intégrées, et ce depuis la Pologne des partages, la lecture de la pauvreté privilégie des entrées par groupes sociaux et tranches d'âges. Mais par rapport à d'autres États post-socialistes, la Pologne se singularise par un recours constant à l'action des niveaux décentralisés et déconcentrés, un défi qu'a tenté de relever précocement la troisième ville du pays, Łódź.

\section{Lódź au défi des quartiers insalubres et de la pauvreté des enfants}

L'intérêt de cette ville vis-à-vis des approches de la pauvreté est qu'elle est à l'origine de certaines des problématiques déclinées dans les programmes nationaux puis des préconisations politiques, grâce à une mise en évidence empirique par la recherche de terrain. La voïvodie de Łódź (730 000 habitants en 2011) occupe le centre du pays, à environ $120 \mathrm{~km}$ au sud-ouest de la capitale. Elle est dominée par la ville qui lui donne son nom, ville champignon née au $\mathrm{XIX}^{\mathrm{e}}$ siècle grâce à l'industrie textile. C'est donc une ville monoindustrielle, ou du moins c'était, car la perte de marchés pour cette activité depuis 1990 force les acteurs locaux à envisager une reconversion économique (Coudroy de Lille L. ET Wolaniuk, 2005). D'autres villes satellites dans la région présentent le même profil et les mêmes héritages sociaux et morphologiques : une population peu formée, une main-d'œuvre 
féminine difficile à requalifier, une pauvreté chronique depuis plus de vingt ans, et un environnement urbain très dégradé dans les quartiers anciens du centre, insalubres. Łódź a perdu $15 \%$ de ses habitants depuis 1980, notamment dans le centre-ville.

La ville est confrontée à ce qu'évoquent quelques rapports nationaux, et surtout locaux : la culture de la pauvreté, et l'apparition d'une underclass. Depuis l'effondrement en 1990 des marchés du textile, à cause de la perte du débouché naturel des «pays frères », et de l'ouverture des frontières à la concurrence asiatique, des familles entières se trouvent touchées par le chômage. Dans des contextes familiaux où plusieurs générations restent sans travail et sans ressources, les repères sociaux, les habitudes de vie des enfants sont forgées dans ce qui est perçu localement comme l'apparition d'une culture de la pauvreté. Or ce sont des sociologues de l'université de Łódź qui, d'une part, ont mis à l'agenda des politiques publiques nationales le thème des enfants comme premières victimes de la pauvreté, et d'autre part montré l'existence de poches de pauvreté dans la ville, en ayant accès aux fichiers des bénéficiaires de l'aide sociale dès 1996-98 (Warzywoda-Kruszyńska, 1999; WarzywodaKruszyńska et Grotowska-Leder, 1996). Les moyennes urbaines et a fortiori régionales des statistiques ne rendent en effet pas compte des concentrations spatiales de la pauvreté : ainsi la voïvodie de Łódź n'apparaît pas comme la région la plus exposée (voir tableau $n^{\circ} 3$ ), et, en 2001, le taux de risque de pauvreté légale était à peu près égal à la moyenne nationale (qui était de 16\%) ; mais dans 17 quartiers du centre-ville ancien et dégradé, il atteignait plus de 30 \% (Warzywoda-Kruszyńska et al., 2003). Les immeubles du XIX ${ }^{\mathrm{e}}$ siècle, très mal entretenus pendant toute la période socialiste, sous gestion municipale, génèrent des conditions de vie insalubres pour leurs habitants, et la gentrification partielle du centre-ville accélère la concentration spatiale des plus pauvres dans des quartiers stigmatisés, vus dans l'opinion comme des repaires de délinquance et de «pathologies sociales ». Appuyée sur cette expertise scientifique locale, la ville de Łódź a bâti de manière évolutive une stratégie locale de lutte contre la pauvreté conjuguant des actions sur les personnes et d'autres sur l'espace urbain ${ }^{8}$. La politique sociale occupe le troisième poste des dépenses de la ville, après les infrastructures et l'éducation. Elle cible en priorité les enfants, et Łódź affirme être la ville la plus en pointe sur ce sujet parmi les grandes villes polonaises.

Elle est par ailleurs une des premières villes en Pologne à avoir mis en place un programme de revitalisation ${ }^{9}$ : depuis 2004, l'ensemble du centre-ville délimité par la couronne de voies ferrées qui l'entoure fait l'objet d'un programme de réhabilitation des immeubles de brique du $\mathrm{XIX}^{\mathrm{e}}$ siècle et des espaces publics, avec l'aide des fonds européens. Pourtant en 2008, les chercheurs académiques, dix ans après avoir identifié les poches de pauvreté, sont revenus y enquêter, et ont constaté peu d'évolutions positives (Jankowski et Warzywoda-Kruszyńska, 2008). Ils pointent une dispersion de l'aide sociale, insuffisamment concentrée sur les îlots et les populations les plus fragiles. Refondée en 2008 sous le titre de Stratégie sociale de revitalisation (Ville de Łódź, 2009), la politique municipale (citant ces recherches) propose d'appréhender de manière complexe et multidimensionnelle les problèmes du centre-ville en ciblant davantage certains îlots : un quadrilatère de $1 \mathrm{~km}^{2}$ près de la gare, et des poches de pauvreté constituées de petits immeubles hérités de cités patronales textiles du XIX ${ }^{\mathrm{e}}$ siècle. Comme son titre l'indique, cette stratégie associe les problématiques sociales et architecturales; on pourrait même affirmer sans trop forcer le trait que le dosage entre approche architecturale et approche sociale de la revitalisation s'inverse entre les deux versions de 2004 et 2008, au bénéfice de la seconde. Il est vrai qu'entre temps, leurs auteurs reconnaissent que des rapports européens ont pointé les problèmes de pauvreté en Pologne : la

\footnotetext{
${ }^{8}$ Ce chapitre s'appuie sur des entretiens réalisés en avril 2010 à la municipalité de la ville (département des affaires sociales), au centre urbain d'aide sociale et au Bureau pour le Partenariat du Développement et des Fonds structurels de la ville de Łódź.

${ }^{9}$ Uproszczony lokalny program rewitalizacji wybranych terenów środmiejskich oraz pofabrycznych Łodzi na lata 2004-2013 [Programme local simplifié de revitalisation d'une sélection de terrains post-industriels de centre-ville de Lodz pour les années 2004-13].
} 
prise en charge de ce thème au niveau communautaire a, en ce sens, eu des effets tangibles au niveau local. Mais les travaux sociologiques de terrain qui restituent le contenu brut de longs entretiens auprès des personnes des quartiers dégradés ont semble-t-il eu pour effet au niveau local, mais aussi national, de faire toucher du doigt la réalité d'une pauvreté que les statistiques accumulées au niveau communautaire rendaient peut-être abstraite. Si la thématique de l'enfance est très présente dans les PNAI polonais, c'est sans doute en partie grâce à leur mise en évidence au niveau local à Łódź.

Quelles formes prend la mise en œuvre de la politique sociale dans cette ville ? Les Centres d'aide sociale constituent au niveau local, comme dans chaque commune de Pologne, le guichet de la politique sociale. Ils existaient avant 1990, mais leurs missions ont été reformulées, leur public élargi ensuite, à la faveur de la décentralisation et de la loi de 2004 sur la politique sociale. Ils distribuent aux personnes éligibles des aides financières, alimentaires, gèrent l'hébergement d'urgence, les maisons d'aide sociale, le placement des personnes (enfants, mères seules, etc..) dans les foyers ou dans un réseau d'un millier de familles d'accueil. Financés par l'État et le powiat, ils collaborent avec de nombreux partenaires externes. À Łódź, le centre urbain d'aide sociale (CUAS) fonctionne ainsi avec une cinquantaine d'associations, religieuses et laïques, qui disposent de ressources plus solides. Ses financements publics proviennent à $80 \%$ de l'État, le reste provenant de la ville (qui a un statut de powiat dans l'architecture territoriale). Les ressources humaines de cet organisme sont considérables : 620 personnes travaillent pour le CAUS lui-même, mais aussi environ un millier dans les maisons d'aide sociale, et 700 dans les lieux d'hébergement ${ }^{10}$. Les fonds structurels contribuent également à la politique sociale, grâce au Fonds social européen : le Bureau du partenariat pour le développement et les fonds structurels est chargé de coordonner et contrôler l'utilisation des fonds européens sur le territoire de la voïvodie, dont ceux du Fonds Social Européen. La ville de Łódź abrite également le siège du powiat, qui est le principal niveau de gestion de l'aide sociale au-dessus de la commune : le powiat gère les centres d'aide familiale, les maisons d'aide sociale, les maisons de l'enfance et les familles d'accueil, les sans-domicile fixe. Le CUAS applique au niveau local la loi sur l'aide sociale de mars 2004 et la Stratégie de résolution des problèmes sociaux de la ville de Łódź de 2005. De 2004 à 2008, le CUAS de Łódź a pris en charge 80000 personnes : 50000 ont reçu des aides financières, 33400 des aides alimentaires. En outre, le CUAS mène des actions d'aide à la réinsertion professionnelle, pourvoit à la diminution du chômage par des emplois aidés, soutient les personnes handicapées dans leurs démarches de vie quotidienne et d'intégration professionnelle, etc. Le CUAS prend également en charge les personnes devant être relogées pour cause de revitalisation urbaine. Il tente enfin de reconstruire des systèmes de solidarité au niveau du quartier, à l'instar de la « journée des voisins » en 2008, pour lutter contre l'isolement (avec des soutiens de l'UE). Ses multiples missions rendent délicate l'évaluation de ses résultats. Le nombre et le pourcentage de personnes vivant dans des ménages bénéficiaires de l'aide sociale dans la voïvodie à légèrement reculé, passant de 14 $\%$ de la population en 2001 à 5,6 en 2011 (mais il est vrai qu'à l'échelle nationale la baisse a atteint des proportions similaires). La direction du CUAS a conscience de la singularité de la ville dans la géographie nationale de la politique sociale, à la fois par la taille du CUAS et par les initiatives qui y ont vu le jour en matière de politique sociale. Sur le terrain, les acteurs ont bien intériorisé la priorité donnée à l'inclusion active par le travail, mais ils reconnaissent que ce mot d'ordre est difficile à mettre en œuvre auprès des publics des enclaves de pauvreté profonde.

\section{Conclusion}

À la question que nous posions d'une prise en considération de la dimension spatiale dans les politiques publiques de lutte contre la pauvreté en Pologne, nous devons donc apporter une

\footnotetext{
${ }^{10}$ Données 2008 du CAUS.
} 
réponse nuancée. Notons tout d'abord qu'une contingence objective la renforce à partir des années 2000 : il s'agit de la réforme territoriale de l'État. Celle-ci a infléchi la construction d'une connaissance de la pauvreté, car la dimension géographique est devenue nécessaire lorsque la décentralisation de l'État est devenue effective, conférant aux voïvodies la mission d'élaborer des politiques sociales régionales, et aux powiat et aux communes celle de les appliquer : il fallait des mesures, des données spatialisées adaptées à ces niveaux d'action. Ainsi, les bases de données du GUS sur la pauvreté jusqu'au niveau LAU 2 (commune) commencent en 2000, alors qu'auparavant elles n'étaient disponibles qu'au niveau régional.

Néanmoins, parallèlement, on a constaté un affaiblissement de la dimension spatiale des politiques nationales dans les documents stratégiques et les PNAI depuis 2004, sauf, précisément du côté de l'identification des acteurs décentralisés. Mais la lutte contre la pauvreté se réalise au niveau local, et dans le cas que nous avons étudié, on assiste à la construction d'une géographie prioritaire de plus en plus resserrée, sur des poches de pauvreté où se conjuguent les efforts de la politique de revitalisation urbaine et de la politique sociale. Mais, en se demandant pourquoi il n'en va pas de même dans certaines localités, certains affirment que, le plus souvent, les acteurs de terrain ne mobilisent pas l'information statistique locale, n'ont pas recours à l'expertise scientifique, et fonctionnement de manière « intuitive » (Kruszyński et Starzyńska, 2010). C'est pourtant ce niveau très local qui apparaît comme essentiel : depuis 2004, les différenciations inter-régionales diminuent, mais la pauvreté augmente dans des secteurs très circonscrits, ruraux ou intra-urbains : en 2008, dans 80 communes polonaises, plus de $20 \%$ de la population était bénéficiaire de l'aide sociale (plus du double de la moyenne nationale), et même plus de $40 \%$ dans 9 d'entre elles (GUS, 2011).

Le fait de centrer l'étude sur la voïvodie et la ville pilotes de Łódź a permis par ailleurs de voir comment les niveaux communautaire, national et local interagissent dans l'appréhension de la question de la pauvreté ${ }^{11}$. On a pu ainsi mettre en évidence une inspiration des politiques nationales «par le haut» des cadrages communautaires, et «par le bas » de l'expertise empirique locale : d'une part l'UE a dans ce domaine comme dans d'autre imposé une vision, un calendrier, des méthodes d'analyse et d'action, des moyens. D'autre part, la production de connaissances locales a permis de cibler les programmes nationaux d'action, en l'occurrence vers l'enfance, rejoignant en cela une préoccupation européenne, mais avec des arguments empiriques qualitatifs et quantitatifs. Cette question de la surreprésentation des enfants dans la population des pauvres reste dans certaines régions largement ouverte, dans des proportions d'ailleurs très supérieures à ce qui a été décrit à Łódź : là où le chômage structurel plafonne à un niveau de $30 \%$ comme la Varmie-Mazurie, jusqu'à $40 \%$ des enfants vivent dans des ménages bénéficiaires de l'aide sociale (GUS, 2011). Or le simple fait de voir émerger en bonne place la question de l'enfant à l'agenda des politiques publiques nationales est en soi une bonne chose, tant cette population «muette » politiquement est invisible le plus souvent pour la classe politique.

Bibliographie

BESKID L., Warunki i sposób życia spoleczeństwa polskiego w sytuacji regresu [Conditions et modes de vie de la société polonaise en situation de régression], Varsovie, Wydawnictwo IFiS PAN, 1987.

COLAS D., L'Europe post-communiste, Paris, PUF, 2002.

COUdROY DE LILlE L., WolANiUK A., «Łódź, ou les ressources territoriales d'une stratégie métropolitaine», Géocarrefour, vol.80,n²1, 2005, p. 35-48.

\footnotetext{
${ }^{11}$ L'équipe de sociologues de Łódź est d'ailleurs solidement impliquée dans les programmes européens sur la pauvreté comme WZLOT ou PROFIT (Policy Responses overcoming factors in the intergenerational transmission of inequalities).
} 
COUdROY DE LILLE L., « Les nouveaux territoires polonais », JEAN Y., BAUDELLE G., L'Europe, aménager les territoires, Paris, A. Colin, 2009, p. 268-281.

DOMANSKI H., Ubóstwo w społeczeństwach post-komunistycznych [La pauvreté dans les sociétés post-communistes], Varsovie, Instytut Spraw Publicznych, 2002.

FRACKIEWICZ L., Sfery niedostatku [Les poches de pauvreté], Varsovie, IWZW, 1983.

JANKOWSKI B., WARZYWODA-KRUSZYNSKA W., 2008, Mieszkańcy tódzkich enklaw biedy 10 lat później [Les habitants des enclaves de pauvreté à Łódź 10 ans après], Łódź, Uniwersytet Łódzki, $57 \mathrm{p}$.

JAROSZ M., Nierówności społeczne [Les inégalités sociales], Varsovie, Książka i wiedza, 1984.

KrUSZYŃSKi K., STARZYŃSKa D., «Zasięg biedy w Polsce. Perspektywa porównacza [L'étendue de la pauvreté en Pologne. Perspective comparative]», Praca socjalna, juilletaoût, 2010, p. 21-50.

MiZEJEWSKI C., «Społeczeństwo dwóch prędkości [Une société à deux vitesses]», Nowe życie gospodarcze, $\mathrm{n}^{\circ} 13$ mars 2005, 2005 p. 4-5.

WARZYWODA-KRUSZYŃSKA W., «Wielkomiejscy biedni - formująca się underclass ?

Przypadek klientów pomocy społecznej [Les pauvres de la grande ville : une underclass en formation ? Le cas des clients de l'aide sociale]», Kultura $i$ spoleczeństwo, vol.42,n ${ }^{\circ}$, 1999, p. 35-52.

WARZYWODA-KRUSZYNSKA W., GROTOWSKA-LEDER J., Wielkomiejska bieda w okresie transformacji (zasiłkobiorcy pomocy społecznej) [La pauvreté des grandes villes en période de transformation (les bénéficiaires de l'aide sociale)], Łódź, WUŁ, 1996.

WARZYWODA-KRUSZYŃSKA W., GROTOWSKA-LEDER J., KRZYSZKOWSKI J., Lokalna polityka wobec biedy i pomocy społecznej [La politique locale envers la pauvreté et l'aide sociale], Łódź, WUŁ, 2003.

WĘCŁAWOWICZ G., «Przestrzeń ubóstwa - nowy wymiar zróżnicowania społecznoprzestrzennego w miastach Polski ? [L'espace de la pauvreté - Nouvelles ou anciennes différenciations socio-spatiales dans les villes polonaises ?]», Przegląd Geograficzny, vol.73, nº 4, 2001, p. 451-475.

WeClawowicz G., Geografia społeczna miast [Géographie sociale des villes], Varsovie, PWN, 2003.

\section{Rapports}

GolinowsKa S., 2004, Narodowa Strategia Integracji Społecznej dla Polski [Stratégie nationale d'intégration sociale pour la Pologne], Varsovie, Ministère de l'Economie, du Travail et de la Polique Sociale, $90 \mathrm{p}$.

GUS, 1997, Sfera ubóstwa w Polsce w świetle badań gospodarstw domowych [Les poches de pauvreté en Pologne vues par les études- ménages], Varsovie, GUS.

GUS, 2003, Sytuacja bytowa gospodarstw domowych w 2003 r [Les conditions de vie des ménages en 2003]., Varsovie, GUS, Wydział Warunków Życia Ludności.

GUS, 2006, Zasięg ubóstwa materialnego w 2005 r. [L'étendue de la pauvreté matérielle en 2005], Varsovie, Główny Urząd Statystytczny.

GUS, 2011, Beneficieni pomocy społecznej i świadczeń rodzinnych [Les bénéficiaires de l'aide sociale et des allocations familiales en 2010], Cracovie,137 p.

GUS, 2012, Różne oblicza polskiej biedy [Les figures de la pauvreté polonaise], Varsovie, Główny Urząd Statystyczny.

Kende P., STRMisKa Z., Egalités et inégalités en Europe de l'est, Paris, PFNSP,1984.

ŁYSON P., 2011, Dochody $i$ warunki życia ludności Polski.(Raport z badania EU-SILC 2009) [Revenus et conditions de vie de la population polonaise (rapport issu de l'étude UE-SILC de 2009)], Varsovie, Główny Urząd Statystyczny. 
ND, 2004, Krajowy Plan Działań na rzecz Integracji Społecznej na lata 2004-2006 [Plan national d'actions pour l'intégration sociale pour les années 2004-06], Varsovie, Ministerstwo Polityki Społecznej.

ND, 2005, Strategia polityki społecznej na lata 2007-13 [Stratégie de la politique sociale pour les années 2007-13], Varsovie, Ministerstwo Polityki Społecznej.

ND, 2006, Krajowy program "Zabezpieczenie społeczne i integracja społeczna na lata 20062008" [Programme national "Sécurité sociale et inclusion sociale pour les années 20062008»], Varsovie, Ministerstwo Pracy i Polityki Społecznej.

ND, 2008, Krajowy program «Zabezpieczenie społeczne i integracja społecna na lata 2008 2010» [Programme national «Sécurité sociale et intégration sociale pour les années 2008-2010»], Varsovie, Ministerstwo Pracy i Polityki Społecznej.

SZUKIEŁOJĆ-BIEKUŃSKA A., 2010, Ubóstwo w Polsce na tle krajów UE w świetle Europejskiego badania dochodów $i$ warunków życia-EU-SILC 2008 [La pauvreté en Pologne par rapport aux pays de l'UE, vue par l'étude européenne sur les revenus et les conditions de vie UE-SILC 2008], Varsovie, Główny Urząd Statystyczny.

SZUKIEŁOJĆ-BIEKUŃSKA A., 2011a, Ubóstwo w Polsce (na podstawie badania budżetów gospodarstw domowych) [La pauvreté en Pologne (d'après l'étude des budgets des ménages)], Varsovie, Główny Urząd Statystyczny.

SzUKIEŁOJĆ-BIEKUŃSKA A., 2011b, Ubóstwo w Polsce w 2011 r. [La pauvreté en Pologne en 2011], Varsovie, Główny Urząd Statystyczny.

VILLE DE ŁóDŹ, 2009, Społeczna Strategia Rewitalizacja [Stratégie sociale de revitalisation], Łódź, Urząd Miasta Łodzi. 